\title{
Percepción de las estrategias que emplean los profesores para mantener la disciplina, razones de los alumnos para ser disciplinados y comportamiento en educación física
}

\section{Perceived teachers' strategies to sustain discipline, pupils' reasons for being disciplined, and pupils' behavior in physical education}

\author{
Melchor Gutiérrez Sanmartín \\ Esther López \\ Universidad de Valencia
}

\begin{abstract}
Resumen
Este trabajo ha relacionado la percepción de las estrategias de los profesores para la disciplina, las razones de los alumnos para ser disciplinados y su comportamiento en educación física (EF). Participaron 2189 adolescentes de 13 a 17 años, que respondieron cuestionarios sobre estrategias para mantener la disciplina, razones para la disciplina y disciplina-indisciplina en EF. La edad y el género de los alumnos son variables diferenciadoras, mientras que la interacción género de los alumnos $x$ género de los profesores no aporta diferencias significativas. Los análisis de regresión han mostrado que las razones intrínsecas de los alumnos y de los profesores y las razones responsables y de preocupación por los demás de los alumnos, predicen la disciplina en EF. Por el contrario, los predictores más importantes de la indisciplina de los alumnos han sido la indiferencia del profesor y la falta de razones de los alumnos para ser disciplinados. Se discuten los resultados en el marco de la teoría de la autodeterminación y en términos de aportación al proceso de enseñanza-aprendizaje de los adolescentes.
\end{abstract}

Palabras clave: adolescentes; educación física; teoría de la autodeterminación; disciplina.

\begin{abstract}
The purpose of this study was to analyze the relationships among perceived teachers' strategies to maintain discipline, pupils' reasons for being disciplined and pupils' behavior in physical education (PE). A sample of 2189 Spanish adolescents, ages 13 to 17 years, responded questionnaires about strategies to sustain discipline, reasons for being disciplined and self-reported discipline in PE lessons. The pupils' age and gender were variables that determined the results, while the interaction pupils' by teachers' gender did not generate significant differences. The regression analyses show that the better predictors of pupils' discipline were the pupils' and teachers' intrinsic reasons, and the pupils' caring and responsibility reasons for being disciplined. On the contrary, the most important predictors of pupils' indiscipline were the teachers' indifference and pupils' no reasons for being disciplined. Results are discussed in the self-determination theory framework and practical issues to adolescents' teaching-learning process.
\end{abstract}

Key words: adolescents; physical education; self-determination theory; discipline.

Correspondencia/correspondence: Melchor Gutiérrez Sanmartín

Dpto. de Psicología Evolutiva y de la Educación. Fac. de Ciencias de la A.F. y el Deporte

Universidad de Valencia, España

E-mail: Melchor.Gutierrez@uv.es 
Gutiérrez, M.; López, E. (2011). Percepción de las estrategias que emplean los profesores para mantener la disciplina, razones de los alumnos para ser disciplinados y comportamiento en educación física. Revista Internacional de Ciencias del Deporte, 22(7), 24-38. http://www.cafyd.com/REVISTA/02203.pdf

\section{Introducción}

$\mathrm{H}$ ace ya un tiempo que las funciones de la educación física (EF) se vienen considerando desde perspectivas diferentes. Mientras unos ponen el énfasis en atribuirle valor en sí misma, por su capacidad para producir sensaciones intrínsecas de bienestar, otros destacan su importancia como medio para conseguir diversos beneficios para la persona. Entre éstos cabe citar: la adquisición de una buena condición física, la incorporación de estilos de vida saludables, la prevención de riesgos para la salud, y el desarrollo de virtudes personales y para la socialización infantil y juvenil (Gutiérrez y Ruiz, 2009; Pérez-Samaniego, Iborra, Peiró y Beltrán, 2010). Estas perspectivas se encuentran en consonancia con las dimensiones "educación en movimiento" y "educación a través del movimiento" anunciadas por Arnold (1991). Por estas razones, resulta de suma actualidad estudiar las condiciones en que se desarrolla la EF, debiendo prestar atención a diversas variables que han demostrado su importancia en el proceso de enseñanza-aprendizaje. Entre estas variables se encuentran las estrategias para la disciplina que emplean los profesores y el comportamiento de los alumnos en clase. Gotzens; Castelló; Genovard, y Badía (2003) y Gutiérrez; López, y Ruiz (2009) señalaban que la disciplina escolar se ha convertido en uno de los núcleos aglutinadores de la preocupación educativa, entre otras razones porque muchos profesores piensan que una clase disciplinada es uno de los indicadores más importantes de la enseñanza exitosa. Esta opinión también la comparten autores como Parker (1995), Bekiari; Kokaridas, y Sakellariou (2006), Muñoz; Pérez, y Martín (2006), Moreno; Cervelló; Martínez, y Alonso (2007), y Wang; Selman; Dishion, y Stormshak (2010), entre otros.

Sin embargo, a pesar de la importancia que psicólogos y educadores le han atribuido a este tema, y de la considerable cantidad de literatura producida con relación al contexto educativo en general, es más bien escasa la relativa al campo concreto de la EF. Bien es cierto que en la última década se han desarrollado diversas investigaciones que han ido ahondando en el papel de la disciplina en las clases de EF, estudios en los que se ha considerado como marco general de justificación científica las teorías motivacionales y la importancia de los ambientes de aprendizaje. A partir del trabajo seminal de Papaioannou (1998), se han ido publicando otros estudios, tanto en el ámbito internacional como en nuestro propio contexto español, acerca de la disciplina en EF (Cervelló, Jiménez, del Villar, Ramos y Santos-Rosa, 2004; Gutiérrez, Ruiz y López, 2010; Gutiérrez et al., 2009; Martínez-Galindo, Alonso, Cervelló y Moreno, 2009; Moreno, Cervelló, Martínez y Ruiz, 2008; Spray, 2002; Spray y Wang, 2001; Zounhia, Hatziharistos y Emmanouel, 2003). Todos estos trabajos han contemplado, de uno u otro modo, alguna de las variables relacionadas con las estrategias que emplean los profesores para mantener la disciplina, las razones que los alumnos argumentan para comportarse adecuadamente, y su actuación disciplinada o indisciplinada en las clases de EF.

El tema de la disciplina ha sido analizado por diferentes investigadores fundamentados en teorías pedagógicas, psicológicas y sociales (Gutiérrez et al., 2009; Lavay, French y Henderson, 1997; Papaioannou, 1998; Zounhia et al., 2003), y la mayoría de ellos han asociado la disciplina con el interés y la motivación por el aprendizaje (Harrison, Blakemore, Buck y Pellet, 1996; Hellison y Templin, 1991). Papaioannou (1998) estudió el tema de la disciplina en EF basado en la Teoría de la Evaluación Cognitiva (CET; Deci y Ryan, 1985, 1991) y el Modelo de Enseñanza de la Responsabilidad Personal y Social de Hellison (TPSRM; Hellison, 1995).

Según la CET, la motivación intrínseca tiene lugar cuando la gente se encuentra totalmente autodeterminada y se implica en una actividad por el puro placer de la actividad en sí misma, mientras que la motivación extrínseca se produce cuando la gente se siente menos 
Gutiérrez, M.; López, E. (2011). Percepción de las estrategias que emplean los profesores para mantener la disciplina, razones de los alumnos para ser disciplinados y comportamiento en educación física. Revista Internacional de Ciencias del Deporte, 22(7), 24-38. http://www.cafyd.com/REVISTA/02203.pdf

autodeterminada. En general, las conductas autodeterminadas son de mayor calidad y están relacionadas más positivamente con diversos aspectos del comportamiento humano que cuando las conductas son más controladas y menos autodeterminadas (Deci y Ryan, 1994; Taylor y Ntoumanis, 2007).

Tal como afirman Deci y Ryan (1991), las razones por las que la gente actúa de una cierta manera pueden variar desde razones totalmente externas (p.e. "lo hago porque otros me lo hacen a mí"), hasta razones intrínsecas (p.e., "lo hago porque es divertido"). Adicionalmente, el Modelo de Responsabilidad de Hellison (1995) sugiere la existencia de cinco niveles por los que los alumnos discurren a medida que van adquiriendo su comportamiento responsable: (0) ausencia de responsabilidad, (1) auto-control, (2) implicación y compromiso, (3) autoresponsabilidad, (4) preocupación por los demás. Quedaría un último nivel, el que se refiere a la transferencia de lo aprendido en clase a otros contextos de la vida social y familiar.

También Vallerand et al. (1992) indicaron que la conducta de las personas está regulada por diversas razones: (a) razones intrínsecas, que producen placer cuando se realiza la actividad; (b) razones identificadas, asociadas a valores y metas personales; (c) razones introyectadas, determinadas por presiones internas (culpabilidad, vergüenza o turbación); (d) razones externas (cumplimiento de reglas, miedo al castigo o refuerzos externos; y (e) ausencia de razones o sin razón (sentimientos de falta de control). Los alumnos sienten mayor autodeterminación cuando las conductas están reguladas por razones intrínsecas y responsables, y sienten menor autodeterminación cuando están reguladas por razones externas o no existen razones que las justifiquen. Las razones que promueven un buen comportamiento en el contexto educativo son las intrínsecas y de responsabilidad, porque permiten a los alumnos sentir que actúan bajo su propia autodeterminación, más que por las normas impuestas o por el miedo a ser castigados (Deci y Ryan, 1991; Spray, 2002; Zounhia et al., 2003).

Papaioannou (1998) clasificó las razones de los alumnos para comportarse adecuadamente en seis categorías: (1) razones intrínsecas, (2) razones externas, (3) razones introyectadas, (4) razones de responsabilidad, (5) razones de preocupación por los demás, y (6) sin razones. En esa investigación, Papaioannou analizó las razones de los alumnos para ser disciplinados, la percepción de las estrategias empleadas por los profesores para mantener la disciplina, las orientaciones de meta, y la percepción del clima motivacional. Sus resultados mostraron que los profesores que ayudaban a sus alumnos a adoptar razones autodeterminadas para comportarse adecuadamente, gozaban de un mayor orden en sus clases.

Con respecto a las estrategias que los profesores emplean frente a los problemas de disciplina, según Zounhia et al. (2003), hay dos categorías. La primera de ellas se refiere a las estrategias basadas en la noción de que los profesores pueden mantener el orden en sus clases mediante la recompensa de las conductas apropiadas y la prevención o el castigo de las conductas inapropiadas (Miller, Ferguson y Simpson, 1998; Siedentop, 1995; Tulley y Chiu, 1998; Wan y Salili, 1996). Estas estrategias, según la CET, promueven razones externas para comportarse adecuadamente en clase. La segunda categoría hace referencia a las estrategias que ayudan a los alumnos a adquirir responsabilidad en su comportamiento, es decir, a incrementar su autodeterminación (Hellison, 1995; Lavay et al., 1997).

Como complemento a los anteriores trabajos, Zounia et al. (2003) desarrollaron un estudio con adolescentes griegos en el que analizaron las razones de los alumnos para comportarse adecuadamente en diferentes materias del currículo escolar y la percepción de las estrategias usadas por los profesores para mantener la disciplina. También analizaron si existía relación entre las razones de los alumnos para ser disciplinados y su percepción de las estrategias 
Gutiérrez, M.; López, E. (2011). Percepción de las estrategias que emplean los profesores para mantener la disciplina, razones de los alumnos para ser disciplinados y comportamiento en educación física. Revista Internacional de Ciencias del Deporte, 22(7), 24-38. http://www.cafyd.com/REVISTA/02203.pdf

empleadas por los profesores para mantener la disciplina en las clases. Los resultados de este trabajo prestaron apoyo a los obtenidos por Papaioannou (1998) y Spray (2002), indicando que las razones para comportarse los alumnos adecuadamente fueron generalmente autodeterminadas, aunque aparecieron diferentes grados de autodeterminación en las distintas asignaturas. Los profesores usaron, en general, estrategias de enseñanza que destacaban las razones intrínsecas para el buen comportamiento, aunque con algunas diferencias según las diferentes materias escolares. Por otro lado, también encontraron que las razones de los alumnos para ser disciplinados estaban significativamente relacionadas con sus percepciones sobre las estrategias que empleaban sus profesores para mantener la disciplina. Al final de su trabajo, Zounhia et al., sugerían realizar investigaciones similares a la suya, en otros países, para conocer mejor qué pasa con la disciplina en diferentes sistemas educativos.

Por ello, fundamentados en el marco teórico expuesto, el objetivo de este trabajo ha pretendido dar un paso más y conocer no sólo las relaciones entre la percepción de las estrategias que emplean los profesores para mantener la disciplina y las razones de los alumnos para ser disciplinados, sino también cuáles de estas variables ofrecen mayor capacidad predictiva del comportamiento (disciplina-indisciplina) de los alumnos en las clases de EF. A la vista de las referencias ofrecidas por la literatura especializada, hipotetizamos una relación positiva entre las estrategias de los profesores para mantener la disciplina fundamentadas en razones intrínsecas, las razones intrínsecas y responsables de los alumnos para ser disciplinados, y su comportamiento disciplinado en EF. Por el contrario, las percepciones de estrategias de los profesores para la disciplina fundamentadas en razones introyectadas y sin razones, estarán relacionadas con las razones introyectadas, externas y sin razones de los alumnos para ser disciplinados y con su indisciplina en la clase de EF.

\section{Método}

\section{Participantes}

Han constituido la muestra 2189 alumnos de ESO y Bachiller (1106 alumnos, 1083 alumnas), con edades comprendidas entre 13 y 17 años $(M=14.78, S D=1.32)$ que cursaban estudios en 43 centros educativos ( 28 colegios públicos y 15 concertados). Los centros fueron seleccionados mediante muestreo por conveniencia (a través de colaboradores), procurando que fuesen representativos de todo el espectro de centros educativos de la Comunidad Valenciana (España), y atendiendo a las variables de edad, género y condición socioeconómica.

\section{Instrumentos}

Percepción de las estrategias que emplean los profesores para mantener la disciplina en clase. Para medir esta variable se utilizó el cuestionario SSDS (Strategies to Sustain Discipline Scale; Papaioannou, 1998), traducido al castellano por Gutiérrez (2003) y validado para muestras españolas por Gutiérrez et al. (2009).

El SSDS consta de 27 items, agrupados por Papaioannou (1998) en cuatro dimensiones: Énfasis del profesor sobre razones intrínsecas para mantener la disciplina $(\alpha=.93)$, Énfasis del profesor sobre razones introyectadas para mantener la disciplina $(\alpha=.76)$, Indiferencia del profesor para mantener la disciplina $(\alpha=.76)$, y Énfasis del profesor sobre razones externas para mantener la disciplina $(\alpha=.62)$. El cuestionario estaba encabezado por la expresión: "Para mantener la disciplina en clase, mi profesor/a de EF..." Ejemplos de cada uno de los factores son: "Atrae nuestra atención para hacer la clase más interesante", "Nos hace sentir mal con nosotros mismos si no somos disciplinados", "No hace nada por 
Gutiérrez, M.; López, E. (2011). Percepción de las estrategias que emplean los profesores para mantener la disciplina, razones de los alumnos para ser disciplinados y comportamiento en educación física. Revista Internacional de Ciencias del Deporte, 22(7), 24-38. http://www.cafyd.com/REVISTA/02203.pdf

mantener la disciplina", "Amenaza con que tendremos problemas si nos portamos mal". Las respuestas se dieron en una escala Likert de 5 puntos, desde (1) Totalmente en desacuerdo, hasta (5) Totalmente de acuerdo.

Mediante Análisis Factorial Confirmatorio (AFC), Gutiérrez et al. (2009) obtuvieron moderados índices de ajuste al modelo propuesto por Papaioannou $(1998)\left(\chi_{272}^{2}=1123.29\right.$, $p<.001$, CFI $=.90$, GFI $=.83$, AGFI $=.90$; SRMR $=.06$, RMSEA $=.04$ ), aunque encontraron únicamente tres dimensiones o factores: énfasis del profesor sobre Razones Intrínsecas para mantener la disciplina; énfasis del profesor sobre Razones Introyectadas; Indiferencia del profesor para mantener la disciplina.

Razones para ser disciplinados en clase. Se empleó una adaptación de la Reasons for Dicipline Scale (RDS; Papaioannou, 1998), traducida al español por Gutiérrez (2003). Con los 26 items que componen esta escala, Papaioannou (1998) obtuvo seis dimensiones: Razones Intrínsecas para ser disciplinado $(\alpha=.90)$, Razones Externas $(\alpha=.76)$, Sin Razones $(\alpha=.68)$, Razones Introyectadas $(\alpha=.72)$, Razones de Responsabilidad $(\alpha=.77)$, y Razones de Preocupación por los demás $(\alpha=.80)$.

El encabezado para todos los ítems fue "Cuando soy disciplinado en clase de EF es porque...". Las respuestas fueron dadas en una escala Likert desde (1) Totalmente en desacuerdo hasta (5) Totalmente de acuerdo. Como ejemplos de cada factor se incluyen los ítems: "Quiero seguir la clase", "Son las reglas", "Siento que pierdo el tiempo siendo disciplinado en EF", "Me fastidia cuando no lo hago", "Procuro ser una persona responsable", "Quiero que mis compañeros comprendan lo que están haciendo correcto o incorrecto".

Mediante AFC, sometimos a comprobación todos los modelos que ofrecía la literatura especializada sobre validación de la RDS: el de Papaioannou (1998), el de Spray y Wang (2001), y el de Moreno et al. (2008). De todos ellos, el que mejores índices de ajuste proporcionó fue el modelo propuesto por Papaioannou $(1998)\left(\chi_{215}^{2}=799.06, p<.001, \mathrm{CFI}=\right.$ .91 , GFI $=.90$, AGFI $=.89$; SRMR $=.08$, RMSEA $=.03)$, con las seis dimensiones propuestas por ese autor.

Comportamiento en clase de EF. Para medir el comportamiento de los alumnos en la clase de EF, se utilizó el Inventario de Conductas de Disciplina-Indisciplina en EF (ICDIEF; Cervelló et al., 2004). Este instrumento está compuesto por 18 ítems, 9 de ellos agrupados en el factor Disciplina $(\alpha=.78)$, y otros 9 agrupados en el factor Indisciplina $(\alpha=.77)$. Ejemplos de items que componen estos factores: "Cumplo las normas que se establecen en el funcionamiento de la clase", "Ayudo a mis compañeros/as cuando lo necesitan", "No atiendo a las explicaciones del profesor/a", "Realizo actividades diferentes a las que el profesor/a me ha propuesto practicar".

Se pidió a los alumnos que respondieran en función de lo que sucedía en sus clases de EF. El encabezado de los ítems era "En las clases de EF...". Las respuestas fueron dadas en una escala Likert desde (1) Totalmente en desacuerdo hasta (5) Totalmente de acuerdo.

Aunque la RDS ya había sido validada por Cervelló et al. (2004), sometimos a verificación mediante AFC su estructura factorial con la muestra estudiada, obteniendo razonables índices de ajuste al modelo propuesto por Cervelló et al. $\left(\chi^{2}{ }_{134}=418.53, p<.001\right.$; CFI $=.97$; GFI $=$ $.91 ; \mathrm{AGFI}=.89 ; \mathrm{SRMR}=.09 ; \mathrm{RMSEA}=.03)$, ratificándose las dos dimensiones anunciadas por sus autores: Disciplina, Indisciplina. 
Gutiérrez, M.; López, E. (2011). Percepción de las estrategias que emplean los profesores para mantener la disciplina, razones de los alumnos para ser disciplinados y comportamiento en educación física. Revista Internacional de Ciencias del Deporte, 22(7), 24-38. http://www.cafyd.com/REVISTA/02203.pdf

\section{Procedimiento}

A partir de un foro en el que participaban profesores de EF de diversos centros educativos, se les solicitó colaboración para desarrollar la investigación, y además se les propuso el contacto con otros compañeros que también pudieran estar dispuestos a colaborar. Posteriormente, se escribió a los directores de los centros en los que trabajaban estos profesores, para explicarles la investigación y solicitar permiso para realizarla. Una vez obtenido el consentimiento de las autoridades de los centros y de los profesores colaboradores, se enviaron cartas a los padres de los alumnos solicitándoles permiso para la participación de sus hijos, por ser éstos menores de edad. Los instrumentos fueron aplicados por un miembro del equipo de investigación, sin la presencia del profesor de EF en el aula, para facilitar la espontaneidad en las respuestas de los alumnos. Los datos se recogieron entre los meses de abril y mayo para garantizar que alumnos y profesores hubieran podido conocerse mutuamente. Todos los alumnos participaron de forma voluntaria después de haberles explicado los objetivos de la investigación.

\section{Análisis de datos}

Puesto que ya se disponía de soluciones factoriales de todos los instrumentos empleados, obtenidas mediante AFC, se procedió a calcular la fiabilidad de cada una de las dimensiones o factores mediante el coeficiente alfa de Cronbach $(\alpha)$. Seguidamente, se calcularon los estadísticos descriptivos y las correlaciones entre todas las variables estudiadas. También se desarrollaron ANOVAS de un factor para analizar las posibles diferencias en función de la edad de los alumnos, y ANOVAS factoriales $(2 \times 2)$ para analizar las diferencias según el género de los alumnos, el género de los profesores y la interacción género de los alumnos por género de los profesores. Finalmente, se aplicaron análisis de regresión para predecir el comportamiento de los alumnos en las clases de EF.

\section{Resultados}

\section{Análisis descriptivo y correlacional}

En los resultados que muestra la Tabla 1 se observa que la fiabilidad de los factores aportados por los instrumentos utilizados han sido en todos los casos satisfactorios, con valores de $\alpha$ iguales o superiores a .70 (Nunnally y Bernstein, 1995), a excepción del factor "Indiferencia de los profesores para la disciplina", con un $\alpha=.66$. No obstante, se ha mantenido este factor en los análisis en atención a las consideraciones de Ford; MacCallum, y Tayt (1986), que ponen el énfasis en el peso factorial de los reactivos, considerándolos satisfactorios con valores superiores a .40 (en el presente caso se encuentran entre .72 y .85), y dado que el número relativamente pequeño de items en el factor no favorece un valor elevado de alfa.

De los resultados descriptivos que muestra la Tabla 1, cabe destacar la elevada percepción que los alumnos tienen del "énfasis de los profesores en razones intrínsecas para mantener la disciplina" $(M=3.71)$, la importancia que los alumnos atribuyen a las "razones intrínsecas" $(M=3.89)$ así como a las "razones responsables" para ser disciplinados $(M=3.81)$ y su autoinforme de "disciplina" en clase $(M=3.82)$. Por otro lado, también cabe resaltar el bajo nivel de "indisciplina" $(M=1.96)$ del que informan los alumnos.

Con respecto a las correlaciones entre las variables sometidas a estudio (Tabla 1), los resultados más relevantes son los que relacionan la percepción de las estrategias de los profesores fundamentadas en "razones intrínsecas para mantener la disciplina" con cuatro factores de las razones de los alumnos para ser disciplinados: "razones intrínsecas" $(r=.65)$, "razones introyectadas" $(r=.58)$, "razones responsables" $(r=.51)$ y "razones de preocupación por los demás" $(r=.49)$. Cuando los alumnos perciben que sus profesores 
Gutiérrez, M.; López, E. (2011). Percepción de las estrategias que emplean los profesores para mantener la disciplina, razones de los alumnos para ser disciplinados y comportamiento en educación física. Revista Internacional de Ciencias del Deporte, 22(7), 24-38. http://www.cafyd.com/REVISTA/02203.pdf

ponen el énfasis en "razones introyectadas" o culpabilizadoras para mantener la disciplina, esto correlaciona con los factores de los alumnos para ser disciplinados fundamentados en "razones externas" $(r=.28)$, "sin razones" $(r=.27)$ y razones introyectadas" $(r=.38)$. Además, la "indiferencia" de los profesores ante la disciplina correlaciona negativamente con las "razones intrínsecas" de los alumnos para ser disciplinados $(r=-.24)$, positivamente con las "razones externas" $(r=.24)$ y las "sin razones" de los alumnos $(r=.40)$.

Tabla 1. Estadísticos descriptivos, valores de alfa y correlaciones entre la percepción de las estrategias que emplean los profesores para la disciplina, las razones de los alumnos para ser disciplinados y el comportamiento en clase de EF.

\begin{tabular}{|c|c|c|c|c|c|c|c|c|}
\hline Variables & $M$ & $D T$ & $\alpha$ & 1 & 2 & 3 & 10 & 11 \\
\hline \multicolumn{9}{|c|}{ Estrategias de los profesores para mantener la disciplina en clase } \\
\hline 1. Énf en Raz Intrínsecas & 3.71 & 0.6 & .90 & 1.00 & & & & \\
\hline 2. Énf Raz Introyectadas & 2.40 & 0.9 & .72 & $-.17 * *$ & 1.00 & & & \\
\hline 3. Indiferencia Disciplina & 2.22 & 0.8 & .66 & $-.34 * *$ & $.60 * *$ & 1.00 & & \\
\hline \multicolumn{9}{|c|}{ Razones de los alumnos para ser disciplinados en clase } \\
\hline 4. Raz Intrínsecas & 3.89 & 0.7 & .81 & $.65 * *$ & $-.16 * *$ & $-.24 * *$ & $.51 * *$ & $-.20 * *$ \\
\hline 5. Raz Externas & 2.97 & 0.9 & .72 & $.12 * *$ & $.28 * *$ & $.24 * *$ & $.08 * *$ & $.18^{* *}$ \\
\hline 6. Sin Razones & 2.17 & 0.9 & .77 & $-.15 * *$ & $.27 * *$ & $.40^{* *}$ & $-.21 * *$ & $.44^{* *}$ \\
\hline 7. Raz Introyectadas & 3.17 & 0.6 & .70 & $.58 * *$ & $.38 * *$ & $.08 * *$ & $.26 * *$ & $.06^{* *}$ \\
\hline 8. Raz Responsables & 3.81 & 0.6 & .72 & $.51 * *$ & -.04 & $-.16^{* *}$ & $.55 * *$ & $-.27 * *$ \\
\hline 9. R Preoc por los Demás & 3.38 & 0.8 & .76 & $.49 * *$ & .03 & $-.08 * *$ & $.48 * *$ & $-.12 * *$ \\
\hline \multicolumn{9}{|c|}{ Comportamiento de los alumnos en la clase de EF } \\
\hline 10. Disciplina & 3.82 & 0.6 & .75 & $.50 * *$ & $-.13 * *$ & $-.24 * *$ & 1.00 & $-.41 * *$ \\
\hline 11. Indisciplina & 1.96 & 0.8 & .85 & $-.22 * *$ & $.37 * *$ & $.48 * *$ & $-.41 * *$ & 1.00 \\
\hline
\end{tabular}

${ }^{*} p<.05 ; * * p<.01$.

Finalmente, cabe señalar que la "disciplina" de los alumnos se encuentra significativamente relacionada con las "razones intrínsecas" de los profesores para mantener la disciplina $(r=$ $.50)$, las "razones intrínsecas" de los alumnos para ser disciplinados $(r=.51)$, las "razones responsables" ( $r=.55)$ y las "razones de preocupación por los demás" $(r=.48)$. La relación es negativa con el resto de variables. En coherencia con ello, las correlaciones entre la "indisciplina" de los alumnos en el aula y el resto de variables estudiadas muestran la cara opuesta de lo anteriormente especificado para la "disciplina".

\section{Diferencias según la edad de los alumnos}

Según la información que ofrece la Tabla 2, no aparecen diferencias significativas en función de la edad de los alumnos en su percepción de las razones intrínsecas que emplean los profesores para mantener la disciplina en clase. Por el contrario, sí existen diferencias entre los alumnos de 14 años y los de 16 en la percepción de las estrategias que emplean sus profesores con énfasis en razones introyectadas, siendo los alumnos de 16 años quienes las perciben en mayor cantidad. En cuanto a la indiferencia de los profesores ante la disciplina, la perciben en mayor grado los alumnos más jóvenes, y va descendiendo ésta a medida que aumenta la edad.

La edad también resulta ser una variable diferenciadora en todas las razones que los alumnos manifiestan para ser disciplinados en clase, excepto en las razones introyectadas. La 
Gutiérrez, M.; López, E. (2011). Percepción de las estrategias que emplean los profesores para mantener la disciplina, razones de los alumnos para ser disciplinados y comportamiento en educación física. Revista Internacional de Ciencias del Deporte, 22(7), 24-38. http://www.cafyd.com/REVISTA/02203.pdf

tendencia general que muestran los alumnos en todos los casos es que en las edades más jóvenes puntúan más las razones intrínsecas, las razones externas, las sin razones, las razones de responsabilidad y las razones de preocupación por los demás, y estas puntuaciones van descendiendo a medida que aumenta la edad.

Los alumnos de 13 años son los que informan de un comportamiento más disciplinado en clase, frente a los demás grupos de edad. Finalmente, los alumnos de 14 años informan de un mayor grado de indisciplina que el resto de los alumnos.

Tabla 2. Resultados diferenciales según la edad de los alumnos.

\begin{tabular}{|c|c|c|c|c|c|c|c|c|}
\hline Variables & 13 & 14 & 15 & 16 & 17 & $F$ & $p$ & Diferencias entre: \\
\hline \multicolumn{9}{|c|}{ Estrategias de los profesores para mantener la disciplina en clase } \\
\hline Énf Raz Intrínsecas & 3.76 & 3.70 & 3.71 & 3.67 & 3.69 & 1.21 & n.s. & ninguna \\
\hline Énf Raz Introyectadas & 2.46 & 2.47 & 2.40 & 2.30 & 2.32 & 2.88 & * & 14-16 \\
\hline $\begin{array}{l}\text { Indiferencia ante la } \\
\text { Disciplina }\end{array}$ & 2.35 & 2.28 & 2.20 & 2.13 & 2.11 & 7.06 & $* * *$ & $\begin{array}{c}13-15,13-16,13-17 \\
14-16,14-17\end{array}$ \\
\hline \multicolumn{9}{|c|}{ Razones de los alumnos para ser disciplinados en clase } \\
\hline Razones Intrínsecas & 4.03 & 3.87 & 3.87 & 3.84 & 3.82 & 5.84 & $* * *$ & $\begin{array}{c}13-14,13-15,13-16 \\
13-17,14-15\end{array}$ \\
\hline Razones Externas & 3.21 & 3.12 & 2.93 & 2.76 & 2.73 & 23.82 & $* * *$ & $\begin{array}{l}13-15,13-16,13-17 \\
14-15,14-16,14-17\end{array}$ \\
\hline Sin Razones & 2.24 & 2.28 & 2.15 & 2.04 & 2.09 & 5.54 & $* * *$ & $13-16,14-16,14-17$ \\
\hline Razones Introyectadas & 3.18 & 3.21 & 3.19 & 3.11 & 3.12 & 1.81 & n.s. & ninguna \\
\hline Raz de Responsabilidad & 3.90 & 3.83 & 3.80 & 3.74 & 3.75 & 5.05 & $* * *$ & $13-16,13-17$ \\
\hline $\begin{array}{l}\text { Razones de Preocupac. } \\
\text { por los demás }\end{array}$ & 3.54 & 3.37 & 3.34 & 3.27 & 3.33 & 7.78 & $* * *$ & $\begin{array}{c}13-14,13-15,13-16 \\
13-17\end{array}$ \\
\hline \multicolumn{9}{|c|}{ Comportamiento de los alumnos en la clase de EF } \\
\hline Disciplina & 3.94 & 3.77 & 3.79 & 3.82 & 3.78 & 6.71 & $* * *$ & $\begin{array}{c}13-14,13-15,13-16, \\
13-17\end{array}$ \\
\hline Indisciplina & 1.91 & 2.07 & 2.00 & 1.88 & 1.91 & 4.89 & $* *$ & $13-14,14-16,14-17$ \\
\hline
\end{tabular}

$*: p<.05, \quad * *: p<.01, \quad * * *: p<.001$, n.s. $=$ no significativo

Diferencias según el género de los alumnos y el de sus profesores

Los resultados del ANOVA factorial 2x2 (género de los alumnos por género de los profesores) que muestra la Tabla 3, indican que el género de los alumnos aporta diferencias estadísticamente significativas en 9 de las 11 variables estudiadas, no encontrándose diferencias entre alumnos y alumnas en las razones intrínsecas y en las razones introyectadas para ser disciplinados. En cuanto a las diferencias encontradas, los alumnos muestran mayores puntuaciones en la percepción de las razones intrínsecas, razones introyectadas e indiferencia ante la disciplina de los profesores, así como también en las razones externas, sin razones y disciplina de los alumnos en clase. Por el contrario, las alumnas puntúan más las razones de responsabilidad, las razones de preocupación por los demás y la indisciplina de los alumnos en la clase de EF.

Considerado el género de los profesores como variable independiente, se obtienen diferencias estadísticamente significativas únicamente en cuatro de las once variables estudiadas, siendo las puntuaciones más elevadas en las clases dirigidas por profesoras: énfasis en razones 
Gutiérrez, M.; López, E. (2011). Percepción de las estrategias que emplean los profesores para mantener la disciplina, razones de los alumnos para ser disciplinados y comportamiento en educación física. Revista Internacional de Ciencias del Deporte, 22(7), 24-38. http://www.cafyd.com/REVISTA/02203.pdf

introyectadas e indiferencia ante la disciplina por parte de los profesores, razones introyectadas para ser disciplinados e indisciplina de los alumnos.

Tabla 3. Resultados diferenciales según el género de los alumnos y de los profesores

\begin{tabular}{|c|c|c|c|c|c|}
\hline Variable dependiente & Efecto & Hombres & Mujeres & $F$ & $p$ \\
\hline \multirow[t]{3}{*}{ Énfasis en Raz. Intrínsecas profesores } & género alumnos & 3.68 & 3.74 & 4.58 & $*$ \\
\hline & género profesores & 3.70 & 3.72 & 0.32 & n.s. \\
\hline & gén.al. x gén.prof. & & & 1.12 & n.s. \\
\hline \multirow[t]{3}{*}{ Énf. en Raz. Introyectadas profesores } & género alumnos & 2.56 & 2.40 & 73.57 & $* * *$ \\
\hline & género profesores & 2.31 & 2.49 & 20.45 & $* * *$ \\
\hline & gén.al. x gén.prof. & & & 0.01 & n.s. \\
\hline \multirow[t]{3}{*}{ Indif. de los profesores ante la Disciplina } & género alumnos & 2.35 & 2.10 & 58.90 & $* * *$ \\
\hline & género profesores & 2.18 & 2.27 & 6.52 & $*$ \\
\hline & gén.al. x gén.prof. & & & 0.01 & n.s. \\
\hline \multirow[t]{3}{*}{ Razones Intrínsecas de los alumnos } & género alumnos & 3.92 & 3.86 & 3.26 & n.s. \\
\hline & género profesores & 3.92 & 3.87 & 2.79 & n.s. \\
\hline & gén.al. x gén.prof. & & & 1.55 & n.s. \\
\hline \multirow[t]{3}{*}{ Razones Externas de los alumnos } & género alumnos & 3.05 & 2.90 & 14.35 & $* * *$ \\
\hline & género profesores & 2.94 & 3.01 & 3.20 & n.s. \\
\hline & gén.al. x gén.prof. & & & 0.06 & n.s. \\
\hline \multirow[t]{3}{*}{ Sin Razones de los alumnos } & género alumnos & 2.31 & 2.03 & 55.89 & $* * *$ \\
\hline & género profesores & 2.16 & 2.18 & 0.40 & n.s. \\
\hline & gén.al. x gén.prof. & & & 0.78 & n.s. \\
\hline \multirow[t]{3}{*}{ Raz. Introyectadas de los alumnos } & género alumnos & 3.19 & 3.14 & 2.77 & n.s. \\
\hline & género profesores & 3.13 & 3.20 & 6.73 & $*$ \\
\hline & gén.al. x gén.prof. & & & 0.09 & n.s. \\
\hline \multirow[t]{3}{*}{ Raz. de Responsabilidad de los alumnos } & género alumnos & 3.75 & 3.87 & 19.62 & $* * *$ \\
\hline & género profesores & 3.81 & 3.81 & 0.01 & n.s. \\
\hline & gén.al. x gén.prof. & & & 0.21 & n.s. \\
\hline \multirow[t]{3}{*}{ Raz. Preoc. por los demás de los alumnos } & género alumnos & 3.34 & 3.42 & 5.81 & $*$ \\
\hline & género profesores & 3.37 & 3.39 & 0.29 & n.s. \\
\hline & gén.al. x gén.prof. & & & 0.53 & n.s. \\
\hline \multirow[t]{3}{*}{ Disciplina de los alumnos } & género alumnos & 3.78 & 3.87 & 15.50 & $* * *$ \\
\hline & género profesores & 3.85 & 3.80 & 3.33 & n.s. \\
\hline & gén.al. x gén.prof. & & & 0.21 & n.s. \\
\hline \multirow[t]{3}{*}{ Indisciplina de los alumnos } & género alumnos & 2.14 & 1.78 & 96.04 & $* * *$ \\
\hline & género profesores & 1.92 & 2.00 & 5.96 & $*$ \\
\hline & gén.al. x gén.prof. & & & 0.03 & n.s. \\
\hline
\end{tabular}

$*: p<.05, * *: p<.01, * * *: p<.001$, n.s. $=$ no significativo 
Gutiérrez, M.; López, E. (2011). Percepción de las estrategias que emplean los profesores para mantener la disciplina, razones de los alumnos para ser disciplinados y comportamiento en educación física. Revista Internacional de Ciencias del Deporte, 22(7), 24-38. http://www.cafyd.com/REVISTA/02203.pdf

Por último, el efecto de la interacción género alumnos-género profesores no ha aportado en ningún caso diferencias estadísticamente significativas (ver Tabla 3).

Predicción del comportamiento de los alumnos en EF

Para predecir el comportamiento de los alumnos en la clase de EF, se aplicaron dos análisis de regresión por pasos tomando como variable dependiente la "disciplina" de los alumnos en clase, en primer lugar, y la "indisciplina" en segundo lugar, y como variables predictoras las estrategias de los profesores para mantener la disciplina y las razones de los alumnos para ser disciplinados. Previamente, se comprobó el cumplimiento de supuestos tales como linealidad, independencia y no-colinealidad, necesarios para llevar a cabo este tipo de análisis con los datos aportados por la muestra estudiada. Las variables predictoras fueron incluidas en los modelos de regresión mediante el método "introducir".

Predicción de la disciplina de los alumnos en clase. Tal como muestra la Tabla 4, se ha obtenido un coeficiente global de determinación de $\mathrm{R}^{2}=.424(F=177.90 ; p<.001)$, lo cual indica que las variables independientes introducidas en el análisis han aportado un $42.4 \%$ de la varianza explicada de la variable dependiente (disciplina). En el primer paso, en el que se introdujeron las estrategias de los profesores para mantener la disciplina, se obtuvo un coeficiente de determinación de $\mathrm{R}^{2}=.261$. La variable con mayor capacidad predictiva de la disciplina de los alumnos en el aula ha sido el énfasis de los profesores en razones intrínsecas para mantener la disciplina $(\beta=.477)$.

Tabla 4. Análisis de regresión para predecir la Disciplina de los alumnos

\begin{tabular}{|l|l|l|l|l|c|}
\hline \multicolumn{1}{|c|}{ Variables } & \multicolumn{1}{|c|}{$\mathrm{R}^{2}$} & $\mathrm{R}^{2}$ ajustado & $\beta$ & $t$ & $p$ \\
\hline Paso 1 & .261 & .260 & & & \\
\hline Énfasis profesor en Razones Intrínsecas & & & .477 & 24.40 & $* * *$ \\
\hline Énfasis profesor en Razones Introyectadas & & & -.001 & -0.04 & n.s. \\
\hline Indiferencia del profesor para la disciplina & & & -.080 & -3.35 & $* *$ \\
\hline Paso 2 & .424 & .421 & & & \\
\hline Énfasis profesor en Razones Intrínsecas & & & .139 & 4.76 & $* * *$ \\
\hline Énfasis profesor en Razones Introyectadas & & & -.015 & -0.64 & n.s. \\
\hline Indiferencia profesor para la disciplina & & & -.050 & -2.25 & $*$ \\
\hline Razones Intrínsecas para ser disciplinados & & & .179 & 7.97 & $* * *$ \\
\hline Razones Externas para ser disciplinados & & & -.006 & -0.31 & n.s. \\
\hline Sin Razones para ser disciplinados & & & -.078 & -4.26 & $* * *$ \\
\hline Razones Introyectadas para ser disciplinados & & & -.021 & -0.83 & n.s. \\
\hline Razones Responsables para ser disciplinados & & & .262 & 12.14 & $* * *$ \\
\hline Razones de Preocupación por los demás & & & .195 & 9.31 & $* * *$ \\
\hline
\end{tabular}

$*: p<.05, * *: p<.01, * * *: p<.001$, n.s. $=$ no significativo

En el segundo paso, en el que se han introducido también las razones de los alumnos para ser disciplinados, se ha producido un incremento en $\mathrm{R}^{2}$ de .163 , es decir, se añade un $16.3 \%$ de explicación de varianza en la predicción de la disciplina de los alumnos. En este segundo paso, los coeficientes de regresión de las variables con mayor capacidad predictiva han sido: 
Gutiérrez, M.; López, E. (2011). Percepción de las estrategias que emplean los profesores para mantener la disciplina, razones de los alumnos para ser disciplinados y comportamiento en educación física. Revista Internacional de Ciencias del Deporte, 22(7), 24-38. http://www.cafyd.com/REVISTA/02203.pdf

las razones responsables de los alumnos para la disciplina $(\beta=.262)$, las razones de los alumnos de preocupación por los demás $(\beta=.195)$, las razones intrínsecas para la disciplina $(b=.179)$, y el énfasis de los profesores en razones intrínsecas $(b=.139)$.

Predicción de la indisciplina de los alumnos en la clase de EF. La Tabla 5, que muestra los resultados de la predicción de la indisciplina de los alumnos en clase, indica que el coeficiente de determinación global es de $\mathrm{R}^{2}=.348(F=129.38 ; p<.001)$, es decir un $34.8 \%$ de la varianza explicada de la variable dependiente "indisciplina". Considerando el primer paso, en el que se introdujeron las estrategias de los profesores para mantener la disciplina, se ha obtenido un valor de $\mathrm{R}^{2}=.245$, obteniéndose un incremento de $\mathrm{R}^{2}=.103$ al añadir el segundo paso.

En el primer paso, las variables con mayor capacidad predictiva de la indisciplina de los alumnos en clase han sido: la indiferencia de los profesores para la disciplina $(\beta=.387)$ y el énfasis de los profesores en razones introyectadas o culpabilizadoras para mantener la disciplina $(\beta=.124)$. Sin embargo, cuando también se introducen en el segundo paso las razones de los alumnos para ser disciplinados, se han obtenido los siguientes coeficientes de regresión, por orden de importancia en la predicción de la indisciplina de los alumnos en clase de EF: la indiferencia de los profesores ante la disciplina $(\beta=.272)$, la falta de razones de los alumnos para ser disciplinados $(\beta=.259)$, las razones responsables de los alumnos para ser disciplinados, en sentido opuesto $(\beta=-.209)$, y el énfasis de los profesores en razones introyectadas para mantener la disciplina $(\beta=.104)$.

Tabla 5. Análisis de regresión para predecir la Indisciplina de los alumnos

\begin{tabular}{|c|c|c|c|c|c|}
\hline Variables & $\mathrm{R}^{2}$ & $\mathrm{R}^{2}$ ajustado & $\beta$ & $t$ & $p$ \\
\hline Paso 1 & .245 & .244 & & & \\
\hline Énfasis profesor en Razones Intrínsecas & & & -.063 & -3.18 & $* *$ \\
\hline Énfasis profesor en Razones Introyectadas & & & .124 & 5.36 & $* * *$ \\
\hline Indiferencia profesor para la disciplina & & & .387 & 15.95 & $* * *$ \\
\hline Paso 2 & .348 & .346 & & & \\
\hline Énfasis profesor en Razones Intrínsecas & & & .007 & 0.24 & n.s. \\
\hline Énfasis profesor en Razones Introyectadas & & & .104 & 4.07 & $* * *$ \\
\hline Indiferencia profesor para la disciplina & & & .272 & 11.41 & $* * *$ \\
\hline Razones Intrínsecas para ser disciplinados & & & .014 & 0.58 & n.s. \\
\hline Razones Externas para ser disciplinados & & & .052 & 2.71 & $*$ \\
\hline Sin Razones para ser disciplinados & & & .259 & 13.29 & $* * *$ \\
\hline Razones Introyectadas para ser disciplinados & & & .032 & 1.21 & n.s. \\
\hline Razones Responsables para ser disciplinados & & & -.209 & -9.11 & $* * *$ \\
\hline Razones de Preocupación por los demás & & & -.003 & -0.12 & n.s. \\
\hline
\end{tabular}

$*: p<.05, * *: p<.01, * * *: p<.001, \quad$ n.s. $=$ no significativo 
Gutiérrez, M.; López, E. (2011). Percepción de las estrategias que emplean los profesores para mantener la disciplina, razones de los alumnos para ser disciplinados y comportamiento en educación física. Revista Internacional de Ciencias del Deporte, 22(7), 24-38. http://www.cafyd.com/REVISTA/02203.pdf

\section{Discusión y conclusiones}

Encuadrado en el marco de la teoría de la evaluación cognitiva y los principios de la autodeterminación, este trabajo ha consistido en analizar las relaciones entre la percepción que los alumnos tienen de las estrategias que emplean sus profesores para mantener la disciplina, sus razones para ser disciplinados y su comportamiento en las clases de EF. Hipotetizamos, por un lado, la existencia de una relación positiva entre las estrategias de los profesores para mantener la disciplina fundamentadas en razones intrínsecas, las razones intrínsecas y responsables de los alumnos para ser disciplinados, y su comportamiento disciplinado en EF. Por otro lado, hipotetizamos también que las estrategias de los profesores para la disciplina fundamentadas en razones introyectadas y sin razones, estarían relacionadas con las razones introyectadas, externas y sin razones de los alumnos para ser disciplinados y con su indisciplina en la clase de EF.

Los resultados de este trabajo dan cumplimiento a las hipótesis planteadas y se encuentran próximos a los ya expresados en la literatura especializada (Cervelló et al., 2004; Zounhia et al., 2003), corroborando asimismo los de Papaioannou (1998), Spray (2002), Spray y Wong (2001), Lewis; Romi; Qui, y Katz (2005) y Martínez-Galindo et al. (2009). Además, en paralelismo con los resultados de Zounhia et al. (2003), han mostrado una correspondencia entre las percepciones de los alumnos sobre las estrategias empleadas por los profesores para mantener la disciplina y las razones para ser disciplinados en clase. Las variables que mayor grado de correlación han presentado, han sido las razones intrínsecas de unos y otros, así como las razones introyectadas de los alumnos y de sus profesores. Cabe resaltar la relación entre las sin razones de los alumnos y la indiferencia de los profesores ante la disciplina. Por otro lado, también son destacables las relaciones entre la disciplina de los alumnos en clase y sus razones fundamentadas en razones intrínsecas para ser disciplinados, razones de responsabilidad y razones de preocupación por los demás. Por el contrario, la indisciplina se relaciona fundamentalmente con la falta de razones para ser disciplinados.

Con respecto a la predicción de la "disciplina" de los alumnos en clase, han sido tanto las razones intrínsecas de los profesores como las de los alumnos las que han ofrecido mayor capacidad predictiva, junto a las razones responsables de los alumnos y su preocupación por los demás. Por el contrario, las variables más capaces de predecir la "indisciplina" de los alumnos han sido la indiferencia de los profesores, la falta de razones de los alumnos y las razones responsables de los alumnos para ser disciplinados, con signo contrario.

De cara a su aplicación práctica, los resultados de este trabajo sugieren la importancia de que los profesores de EF se esfuercen en la creación de climas motivacionales favorecedores de la autodeterminación de los alumnos, por sus efectos sobre el comportamiento en clase, puesto que la literatura especializada ha mostrado que si los profesores promueven la motivación intrínseca y se favorecen actividades y situaciones de autodeterminación en los alumnos, éstos pueden tener mayor éxito en sus resultados escolares. Por ello, los profesores de EF deben utilizar las estrategias necesarias para facilitar la autodeterminación de los alumnos, propiciando que las razones para ser disciplinados se fundamenten más en razones intrínsecas y responsables que en razones asociadas al sentimiento de culpa, al castigo o a razones externas e indiferentes a la disciplina, resultados que avalan los obtenidos por Cervelló et al. (2004) y Martínez-Galindo et al. (2009). Como indicaban Kaplan; Cheen, y Midgley (2002) y Lewis et al. (2005), el tema de la disciplina en clase es importante no sólo por el bien que puede proporcionar, sino también por el daño que puede producir una disciplina inapropiada, puesto que los alumnos que perciben una disciplina razonada se comportan de forma menos 
Gutiérrez, M.; López, E. (2011). Percepción de las estrategias que emplean los profesores para mantener la disciplina, razones de los alumnos para ser disciplinados y comportamiento en educación física. Revista Internacional de Ciencias del Deporte, 22(7), 24-38. http://www.cafyd.com/REVISTA/02203.pdf

disruptiva y más responsable que quienes interpretan que sus profesores aplican una disciplina más coercitiva, ausente de razones o cargada de indiferencia. A su vez, si perciben una disciplina razonada, también ellos encontrarán razones para ser disciplinados en clase, lo cual irá en favor de un desarrollo personal más positivo.

Finalmente, los resultados de este trabajo sugieren que un apropiado clima motivacional de la clase y una disciplina fundamentada en razones intrínsecas y responsables favorecerán la predisposición de los alumnos hacia la práctica física tanto en el contexto escolar como fuera de él. No olvidemos que estas condiciones del ambiente de la clase pueden repercutir favorablemente sobre las actitudes de los alumnos hacia la EF y sus profesores (Gutiérrez, Pilsa y Torres, 2007; Gutiérrez y Ruiz, 2009; Lewis, 2001), generando asimismo unas actitudes más positivas hacia la práctica de actividad física regular (Granda, Barbero y Montilla, 2008; Taylor y Ntoumanis, 2007).

Limitaciones y sugerencias de investigación

Como todos los trabajos, éste también presenta sus limitaciones. Por ejemplo, los datos se han obtenido a través de colaboradores (muestreo por conveniencia), lo cual podría haber incorporado algún sesgo en la representación poblacional. Por otro lado, los datos se han obtenido mediante auto-informes, con la subjetividad que esto puede conllevar. De cara a futuras investigaciones, deberían recogerse no sólo las percepciones de los alumnos y las valoraciones de sus profesores, sino también la observación directa del clima de la clase y del comportamiento de los alumnos, tratando de aprehender una estimación más objetiva del proceso de enseñanza/aprendizaje. Además, deberían contrastarse los resultados obtenidos en EF con los de otras materias escolares, en consonancia con lo desarrollado por Zounhia et al. (2003).

\section{Referencias}

Arnold, P. (1991). Educación física, movimiento y currículum. Madrid: Morata.

Bekiari, A.; Kokaridas, D., \& Sakellariou, K. (2006). Associations of students' self-reports of their teachers' verbal aggression, intrinsic motivation, and perceptions of reasons for discipline in Greek physical education classes. Psychological Reports, 98, 451461.

Cervelló, E.; Jiménez, R.; del Villar, F.; Ramos, L., \& Santos-Rosa, F. (2004). Goal orientation, motivational climate, equality, and discipline of Spanish physical education students. Perceptual and Motor Skills, 99, 271-283.

Deci, E. L., \& Ryan, R. M. (1985). Intrinsic motivation and self-determination in human behavior. New York: Plenum Press.

Deci, E. L., \& Ryan, R. M. (1991). A motivational approach to self: integration in personality. In, R. Dientsbier (Ed.) Nebraska Symposium on Motivation. Perspectives on motivation (pp. 237- 288). Lincoln, NE: University of Nebraska Press.

Deci, E. L., \& Ryan, R. M. (1994). Promoting self-determined education. Scandinavian Journal of Educational Research, 38(1), 3-14.

Ford, J.; MacCallum, R., \& Tayt, M. (1986). The application of factor analysis in psychology: A critical review and analysis. Personnel Psychology, 39, 291-314.

Gotzens, C.; Castelló, A.; Genovard, C., y Badía, M. (2003). Percepciones de profesores y alumnos de ESO sobre la disciplina en el aula. Psicothema, 15, 362-368.

Granda, J.; Barbero, J. C., y Montilla, M. (2008). Orientaciones de meta y compromiso físico-motor en educación física. Un estudio en alumnos de $4^{\circ}$ de Educación Primaria. Revista Internacional de Ciencias del Deporte, 4(11), 29-41. 
Gutiérrez, M.; López, E. (2011). Percepción de las estrategias que emplean los profesores para mantener la disciplina, razones de los alumnos para ser disciplinados y comportamiento en educación física. Revista Internacional de Ciencias del Deporte, 22(7), 24-38. http://www.cafyd.com/REVISTA/02203.pdf

Gutiérrez, M. (2003). Manual sobre valores en la educación física y el deporte. Barcelona: Paidós.

Gutiérrez, M.; Pilsa, C., y Torres, E. (2007). Perfil de la educación física y sus profesores desde el punto de vista de los alumnos. Revista Internacional de Ciencias del Deporte, 3(8), 39-52.

Gutiérrez, M., \& Ruiz, L. M. (2009). Perceived motivational climate, sportsmanship, and students' attitudes toward physical education classes and teachers. Perceptual and Motor Skills, 108, 308-326.

Gutiérrez, M.; López, E., y Ruiz, L. M. (2009). Estrategias para mantener la disciplina en las clases de educación física: Validación de su medida y análisis de la concordancia entre las percepciones de los profesores y las de sus alumnos. Revista Mexicana de Psicología, 26(2), 203-212.

Gutiérrez, M.; Ruiz, L. M., \& López, E. (2010). Perceptions of motivational climate and teachers' strategies to sustain discipline as predictors of intrinsic motivation in physical education. The Spanish Journal of Psychology, 13(2), 597-608.

Harrison, J.M.; Blakemore, C. L.; Buck, M. M., \& Pellet, T. L. (1996) Instructional Strategies for Secondary School Physical Education. Madison: Brown and Benchmark.

Hellison, D. (1995). Teaching responsibility through physical activity. Champaign, IL: Human Kinetics.

Hellison, D. R., \& Templin, T. J. (1991). A reflective approach to teaching physical education. Champaign, IL: Human Kinetics.

Kaplan, A.; Cheen, M., \& Midgley, C. (2002). Classroom goal structure and student disruptive behaviour. British Journal of Educational Psychology, 72, 191-211.

Lavay, B. W.; French, R., \& Henderson, H. L. (1997). Positive behaviour management strategies for physical educators. Champaign, IL: Human Kinetics.

Lewis, R. (2001). Classroom discipline and student responsibility: The students' view. Teaching and Teacher Education, 17, 307-319.

Lewis, R.; Romi, S.; Qui, X., \& Katz, Y. J. (2005). Teachers' classroom discipline and student misbehavior in Australia, China, and Israel. Teaching and Teacher Education, $21,729-741$.

Martínez-Galindo, C.; Alonso, N.; Cervelló, E., y Moreno, J. A. (2009). Perfiles motivacionales y disciplina en clases de educación física. Diferencias según las razones del alumnado para ser disciplinado y la percepción del trato generado por el profesorado en el aula. Cultura y Educación, 21(3), 331-343.

Miller, A.; Ferguson, E., \& Simpson, R. (1998). The perceived effectiveness of rewards and sanctions in primary schools: Adding in the parental perspective. Educational Psychology, 18(1), 55-64.

Moreno, J. A.; Cervelló, E. M.; Martínez, C., \& Ruiz, L. M. (2008). Preliminary construct validation study of the Reasons for Discipline and Strategies to Sustain Discipline Scales in Spanish physical education. International Journal of Hispanic Psychology, 1, 85-97.

Moreno, J. A.; Cervelló, E. ; Martínez, C., y Alonso, N. (2007). Los comportamientos de disciplina e indisciplina en educación física. Revista Iberoamericana de Educación, 44, 167-190.

Muñoz, M. C.; Pérez, D., y Martín, E. (2006). ¿Qué penalizan los docentes?: Análisis de la disciplina a través de los partes de incidencia. Infancia y Aprendizaje, 29(4), 423435.

Nunnally, J. C., y Bernstein, I. J. (1995). Teoría psicométrica. Madrid: McGraw-Hill. 
Gutiérrez, M.; López, E. (2011). Percepción de las estrategias que emplean los profesores para mantener la disciplina, razones de los alumnos para ser disciplinados y comportamiento en educación física. Revista Internacional de Ciencias del Deporte, 22(7), 24-38. http://www.cafyd.com/REVISTA/02203.pdf

Papaioannou, A. (1998). Goal perspectives, reasons for being disciplined and selfreported discipline in physical education lessons. Journal of Teaching in Physical Education, 17, 421- 441.

Pérez-Samaniego, V.; Iborra, A.; Peiró, C., y Beltrán, V. J. (2010). Actitudes hacia la actividad física: Dimensiones y ambivalencia actitudinal. Revista Internacional de Medicina y Ciencias de la Actividad Física y del Deporte, 10(38), 284-301.

Parker, J. (1995). Secondary teachers' views of effective teaching in physical education. Journal of Teaching in Physical Education, 14, 127-139.

Siedentop, D. (1995). Use positive discipline for middle school student. Strategies, 8(8), 5-8.

Spray, C. (2002). Motivational climate and perceived strategies to sustain pupils' discipline in physical education. European Physical Education Review, 8, 5-20.

Spray, C. M., \& Wang, C. K. J. (2001). Goal orientations, self-determination and pupils' discipline in physical education. Journal of Sports Sciences, 19(2), 903-913.

Taylor, I. M., \& Ntoumanis, N. (2007). Teacher motivational strategies and student selfdetermination in physical education. Journal of Educational Psychology, 99, 747-760.

Tulley, M., \& Chiu, L. H. (1998). Children's perceptions of the effectiveness of classroom discipline techniques. Journal of Instructional Psychology, 25(3), 189-197.

Vallerand, R. J.; Pelletier, L .G.; Blais, M.; Briere, N. M.; Senecal, C., \& Vallieres, E. F. (1992). The Academic Motivational Scale: A measure of intrinsic, extrinsic, and amotivation in education. Educational and Psychological Measurement, 52, 10031017.

Wan, F., \& Salili, F. (1996). Perceived effectiveness of reward and punishment strategies by Hong Kong secondary school students. Psychologia: An International Journal of Psychology in the Orient, 39(4), 261-275.

Wang, M. T.; Selman, R. L.; Dishion, T. J., \& Stormshak, E. A. (2010). A tobit regression analysis of the covariation between middle school students' perceived school climate and behavioral problems. Journal of Research on Adolescence, 20(2), 274-286.

Zounhia, K.; Hatziharistos, D., \& Emmanouel, K. (2003). Greek secondary school pupils' perceived reasons for behaving appropriately and perceived teachers' strategies to maintain discipline. Educational Review, 55, 289-303. 